\title{
Spatial information transmission beyond a system's diffraction limit using optical spectral encoding of spatial frequency
}

\author{
S A Alexandrov ${ }^{1}$ and D D Sampson \\ Optical+Biomedical Engineering Laboratory, School of Electrical, Electronic and \\ Computer Engineering, The University of Western Australia, Crawley, WA, 6009, \\ Australia. \\ E-mail: sergey@ee.uwa.edu.au
}

\begin{abstract}
We propose optical spectral encoding of an object's spatial frequencies as a means of transmitting, through a low-numerical aperture optical system, spatial information with an instantaneous spatial frequency bandwidth wider than the optical system's diffraction-limited bandwidth. We validate this new superresolution approach experimentally and demonstrate one of its possible practical implementations - wide-field spectrally encoded imaging that is sensitive to nanometre-scale local variations in the microstructure of centimetre-scale samples.
\end{abstract}

Key words: optical transfer functions, superresolution, spectral encoding, wide field imaging PACS codes: 42.25.Fx, 42.30.Kq, 42.30.Lr, 42.30.Va, 42.50.St, 07.60.Pb, 07.60.Rd

Submitted to Journal of Optics A, Pure and Applied Optics

Superresolution refers to the transmission through an optical system of an object's spatial frequencies with a bandwidth that exceeds the system's diffraction limit [1]. Such superresolution may $[2,3]$ or may not $[4,5,6,7]$ exceed the ultimate diffraction limit set by the wavelength of light. In the latter category, there are several approaches that extract information about a sample's microstructure that is encoded in an optical beam. One example is light scattering spectroscopic imaging [8,9], based on spatially resolved spectral [8] or angular [9] elastic scattering dependencies. Other examples include static speckle in optical coherence tomography [10] and temporal coherence coding [11]. In these approaches, the encoded spatial information is decoupled from the optical system's numerical aperture (NA), making it possible to transmit and detect such information about a sample's microstructure at spatial frequencies that extend beyond the system's diffraction limit.

In this article, we present a new superresolution approach based on optical spectral encoding and decoding of spatial information. In this approach, the sample's spatial frequencies are spectrally encoded by diffraction of broad (spectral)-band, off-axis plane-wave illumination. The diffracted waves are transmitted through a low-NA, narrow-band optical system, and are decoded by wavelength demultiplexing. Such spectral encoding enables the transmitted spatial frequency bandwidth to greatly exceed that of the optical system, permitting the superresolution of a sample's microstructure in a single capture, over the optical system's wide field of view.

Spectral encoding has previously been used to transmit spatial information through optical fibre $[12,13]$. Dispersive elements at the fibre's input and output were used, in turn, to collect all scattered

\footnotetext{
1 Author to whom any correspondence should be addressed.
} 
wavelength components, and decode the spatial location of those transmitted through the fibre, giving a one-to-one mapping of wavelength components to sample locations. In our approach, by contrast, the spatial frequency component, not the spatial location, is spectrally encoded. Each location on the sample is illuminated by all wavelengths in a broad band and wavelength components are one-to-one mapped to sample spatial frequencies. In the following, we describe our scheme, the range of spatial frequencies that it can transmit, its experimental validation, and one practical means of spectral decoding, sufficient to convincingly demonstrate the approach.

According to image formation theory [14], a suitable sample may be considered to behave as a superposition of diffraction gratings with different orientations, periods, and amplitudes. The complex scalar reflectance or transmission profile of such a sample, including one without periodic structure, can be represented as a corresponding Fourier series of sinusoidal waves. Consider a plane illumination wave incident on a sample at angle $\theta$. The wavelength of light diffracted from a local area with coordinates $(x, y)$ at a given angle $\alpha$ in the plane of incidence will depend on the sample spatial frequency magnitude $v_{S}(x, y)[7]$ according to:

$$
\lambda(x, y)=\frac{|\sin \theta-\sin \alpha|}{v_{S}(x, y)} .
$$

It can be seen from (1) that, at each sample location, each diffracted wavelength component encodes the corresponding sample spatial frequency (Fourier) component. The diffracted wave with wavelength bandwidth $\Delta \lambda$ will transmit sample spatial frequencies with bandwidth $\Delta v_{S}$, given by

$$
\Delta v_{S}=\frac{\Delta \lambda|\sin \theta-\sin \alpha|}{\lambda_{\max } \lambda_{\min }}
$$

where $\lambda_{\max }$ and $\lambda_{\min }$ are the maximum and minimum illumination wavelengths, respectively, assuming a top-hat spectrum. The transmitted spatial frequency band can be shifted, according to (1), by changing the incident and/or the collection angle. Equation (2) shows that, in contrast to conventional microscopy, the transmitted sample spatial frequency bandwidth does not depend on the NA of the optical system and, as a result, spatial frequencies beyond the conventional cut-off can be transmitted. Thus, by using this approach, in combination with a suitable decoding scheme, finer details of the object, not resolvable under conventional imaging, can be observed - a form of superresolution. Such spectral encoding of spatial Fourier components has the potential to characterize microstructure with very high accuracy. For example, if the angles $\theta=60^{\circ}, \alpha=0$ are known accurately and the wavelength $\lambda=500 \mathrm{~nm}$ is measured with a modest accuracy of $1 \mathrm{~nm}$, then the accuracy in determining the sample's corresponding local spatial period is $1.2 \mathrm{~nm}$.

Of course, such spatial information is in only one direction and for non-zero incident angle does not contain the low-frequency diffracted components. The full azimuthal range of the sample's twodimensional spatial frequency components can in principle be passed through the optical system, e.g., by changing the azimuthal angle via rotation of the sample, in which case the resulting superresolved image would be similar to that obtained using dark-field microscopy.

Figure 1 is a schematic diagram of the experimental setup used to validate the basic principle of spectral encoding/decoding. The sample was illuminated off-axis by a plane wave generated from a tungsten-halogen lamp (Ocean Optics, Model LS1) using collimating optics and a pinhole (not shown). The collection angle $\alpha$ was selected by a spatial filter (4-mm diameter) in the focal plane of lens L1 (focal length $\mathrm{f}_{1}=380 \mathrm{~mm}$ ). Spectral decoding was performed by a transmission diffraction grating with frequency $v_{D}=100 \mathrm{l} / \mathrm{mm}$ placed in the image plane behind lens $\mathrm{L} 2\left(\mathrm{f}_{2}=380 \mathrm{~mm}\right)$ (figure $1(\mathrm{a})$ ). The 
decoded spatial frequency spectrum in the focal plane of lens $L 3\left(\mathrm{f}_{3}=60 \mathrm{~mm}\right)$ was recorded by a monochrome CCD camera (Roper Scientific, CoolSNAP). The optical system NA was 0.005, with corresponding conventional transmitted spatial frequency bandwidth of $40 \mathrm{l} / \mathrm{mm}$, field of view of $20 \times 15$ $\mathrm{mm}$, and working distance of $35 \mathrm{~cm}$. The sample spatial period $H=1 / v_{S}$ is related to the focal point deflection distance $s$ in the back focal plane of lens L3 by $H=s /\left(v_{D} f_{3}|\sin \theta-\sin \alpha|\right)$. Interestingly, this linear dependence of sample spatial period on deflection distance is the reciprocal of that due to the conventional optical Fourier transform.

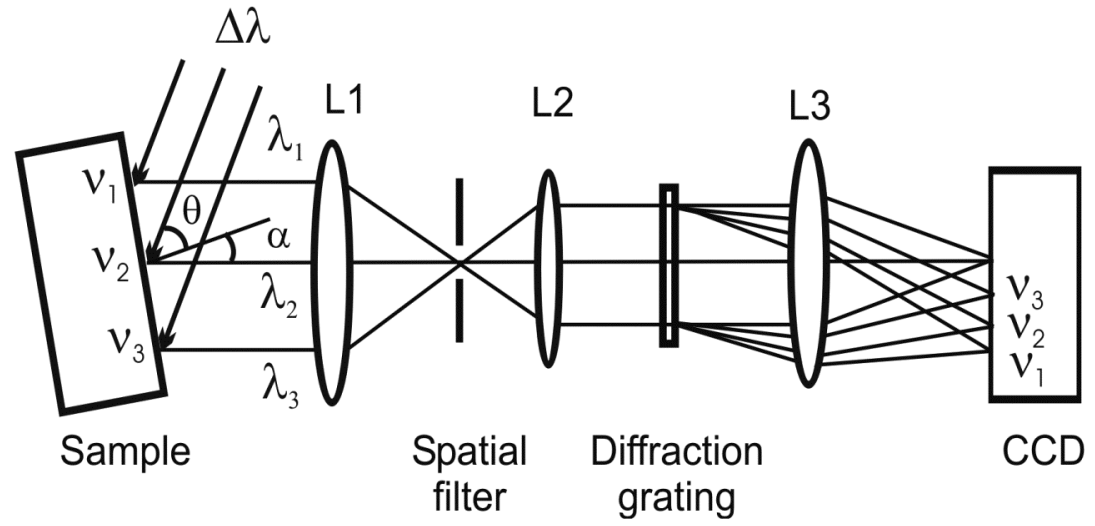

(a)

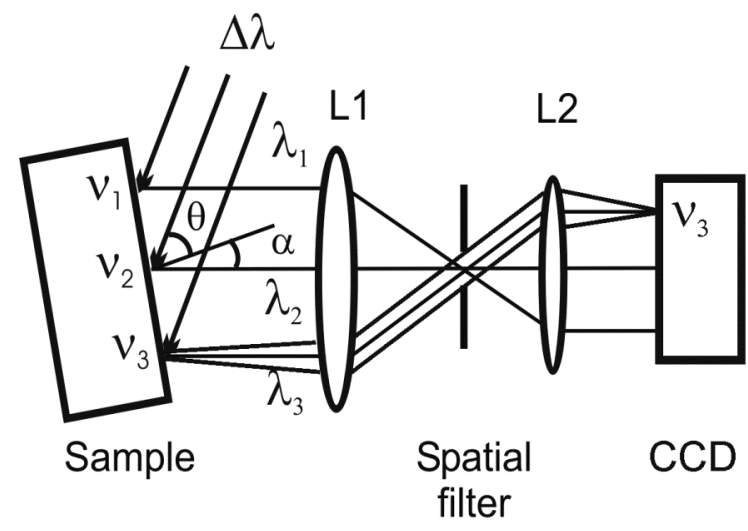

(b)

Figure 1. Schematic diagram of the experimental setup: (a) for validation of the general principle; and (b) for one possible practical implementation - wide-field, spectral-encoded imaging.

To demonstrate the instantaneous transmission of an wide spatial-frequency bandwidth, we employed a composite sample consisting of three reflection diffraction gratings with 1200,830 and 720 grooves $/ \mathrm{mm}$, respectively, and the angles $\theta=30^{\circ}$, and $\alpha=0$. The resulting transmitted bandwidth, 480 $1 / \mathrm{mm}$, is twelve times greater than the diffraction limit of the imaging optics. Note that to resolve the highest frequency grating with a conventional optical system would require a cut-off frequency of greater than $1200 \mathrm{l} / \mathrm{mm}$. Figure 2(a) shows schematically the transfer function for the spectral encoding/decoding experimental optical system and, for comparison, the same system without spectral encoding, under both 
off-axis coherent and incoherent illumination. Figure 2(b) shows the spatial frequency spectrum of the composite sample recorded with a single exposure. The observed spots are convolutions of the transmission profile of the spatial filter and the sample spectrum. The zero-order spot and the three

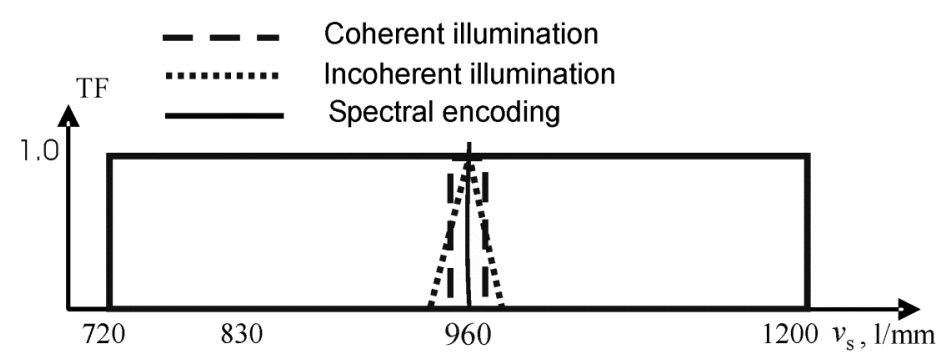

(a)

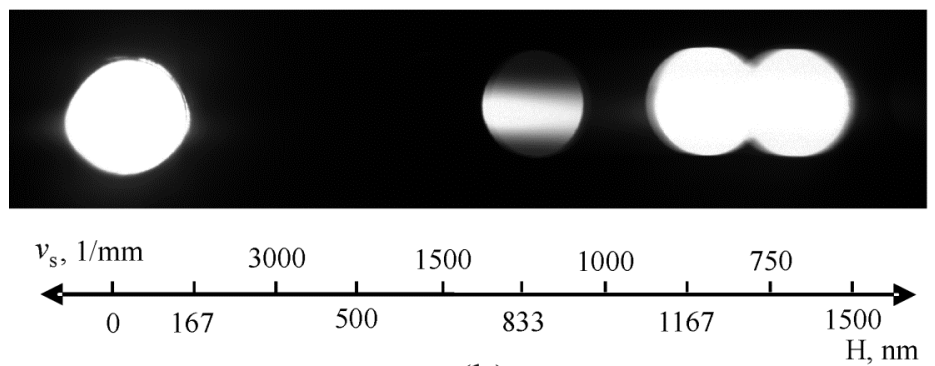

(b)

Figure 2. (a) Transfer functions for the experimental optical system without spectral encoding under coherent and incoherent illumination, and with spectral encoding; (b) The single-exposure decoded sample spatial frequency spectrum versus spatial frequency and period.

first-order spots, corresponding to the spatial frequencies of the three gratings, are clearly seen. Their measured positions agree, to within experimental error, with those calculated from the known optical system parameters. This demonstration validates the superresolution performance of spectral encoding, in simultaneously transmitting one-dimensional spatial frequencies with a bandwidth of twelve times that of the optical system. We know of no other method that could or has achieved such performance.

Having confirmed the validity of the principle, it remains to investigate the extent to which spectral encoding can be used to form a superresolved image. Various means of spectral decoding (i.e., the extraction of the encoded sample spatial frequencies from the transmitted optical spectrum) can be envisaged. We demonstrate one that performs wide-field, single-capture, direct-colour quantitative imaging. In this implementation, the mean superresolved spatial frequency in one direction is measured, and imaged with a modest spatial resolution determined by the low-NA optical system. The decoding grating was replaced with a colour digital camera (JENOPTIK, ProgRes C10plus), and lens L2 was replaced with one of 120-mm focal length (figure. 1(b)). Each colour corresponds to the on-axis (more precisely, averaged within a small solid angle) diffracted component of the sample in a given local area. In this implementation, there is potential ambiguity due to the spectral overlap of different diffraction orders. For absolute measurements, this ambiguity can be avoided by limiting the wavelength range to less than a free spectral range, $\Delta \lambda_{f}=\lambda_{\min } / m$, where $m$ is the diffraction order, corresponding to a spatial frequency bandwidth $\Delta v_{f}=|\sin \theta-\sin \alpha| / m^{2} \lambda_{\max }$. In our experiment, for the visible wavelength range $400-700 \mathrm{~nm}$ and choosing $m=1$, we have $\Delta v=5361 / \mathrm{mm}$ and $\Delta v_{f}=714 \mathrm{l} / \mathrm{mm}$. 
Figure 3 shows images of the composite grating sample, under conventional (non-plane) whitelight illumination and without the spatial filter shown in figure 1 (figure 3(a)), and using spectral encoding (figure 3(b)). Figure 3(b) clearly shows the strong variation in colour associated with the different grating periods, and the periods separated by only $185 \mathrm{~nm}$ are readily distinguished.

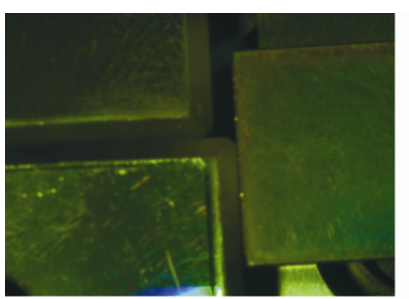

(a)

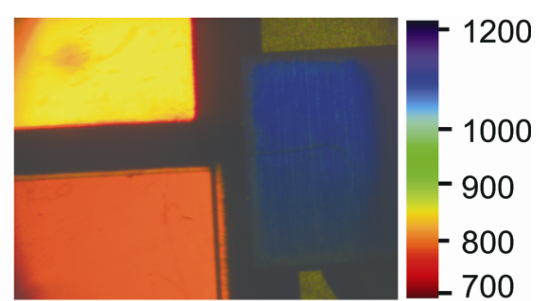

(b)

Figure 3. Images of three gratings: (a) under conventional white-light illumination; and (b) using spectral encoding. Image dimensions are $20 \times 15 \mathrm{~mm}$. The colour scale is in $1 / \mathrm{mm}$.

For the specific means of spectral decoding demonstrated here, the resolved area on the sample is traded off with the resolved microstructure (determined by spectral en/decoding) within it. Such a trade off correctly applies only to this particular implementation and is not a general feature of spectral encoding. The corresponding dependences are:

$$
\begin{gathered}
R_{M}=\frac{\lambda N A}{\sin \theta|\sin \theta-N A|} \\
R_{I}=\frac{0.61 \lambda}{N A},
\end{gathered}
$$

where $N A=n \sin \Delta \alpha$ and $\Delta \alpha=\arctan \left(d / 2 f_{1}\right)$, with $n$ the refractive index of the medium (air) and $d$ the diameter of the spatial filter. As seen from (3) and (4), a decrease in the NA degrades the spatial resolution $R_{I}$ of the wide-field image but simultaneously improves the resolution of the microstructure $R_{M}$. Figure 4 shows the dependence of the microstructure resolution (figure 4 (a)) and the image spatial resolution (figure 4 (b)) on the size of the spatial filter, for the given experimental conditions $\left(\theta=30^{\circ}, \alpha=0^{\circ}\right.$ and $\left.\lambda=500 \mathrm{~nm}\right)$. As is clearly seen from the plots, increasing the size of the spatial filter causes a decrease in the microstructure resolution and an increase in the image spatial resolution.

A parallel can be drawn between the specific means of spectral decoding demonstrated here and phase microscopy techniques. In phase microscopy, the accuracy in determination of the phase or the optical path length (OPL) does not depend on the optical system NA; it merely constrains the minimum area over which the average OPL is measured. The accuracy of the OPL determination is much higher than the spatial resolution of the microscope. In our case, the optical system NA also determines the minimum area over which the mean size of the sample microstructure can be determined, with much higher accuracy than the resolution of the optical system and even higher than the resolution of the best commercially available immersion objective lenses. 


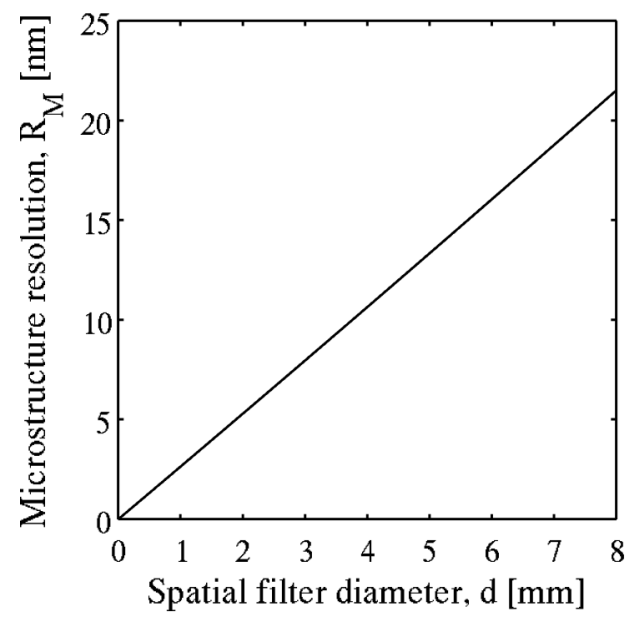

(a)

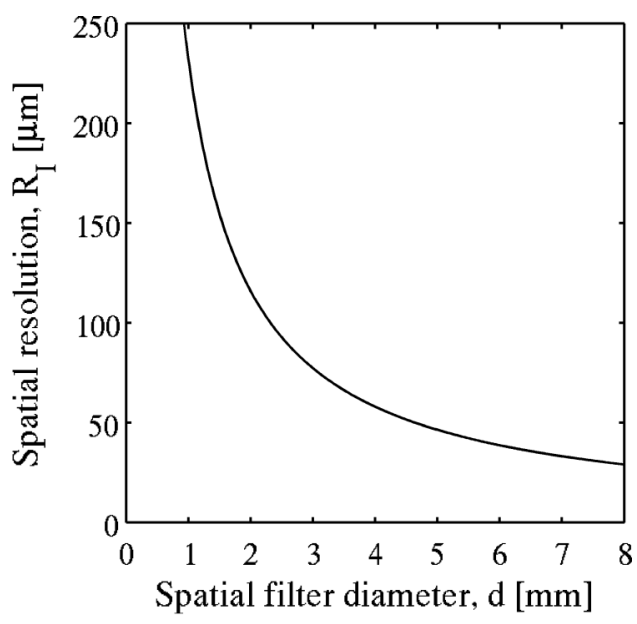

(b)

Figure 4. Dependence of the microstructure resolution and image resolution on the spatial filter diameter for the experimental spectral decoding setup: (a) Microstructure resolution, $R_{M}$; and (b) Spatial resolution of the image, $R_{I}$.

As an example of a more complex sample, figure 5 shows images of a portion of a 1992 Intel Pentium CPU under conventional white-light illumination (figure 5(a)) and using spectral encoding

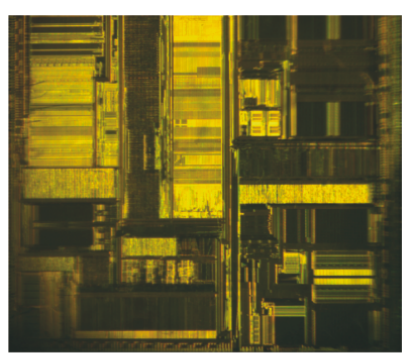

(a)

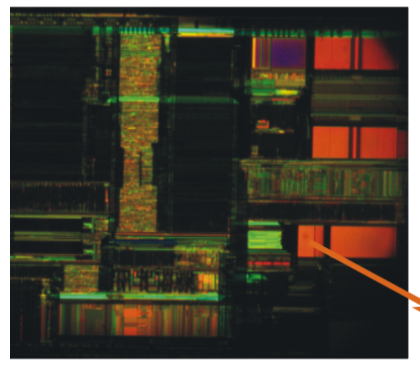

(c)

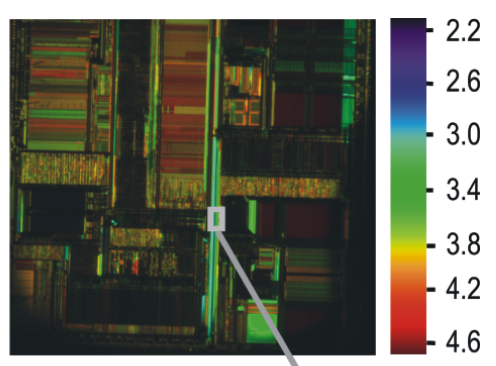

(b)

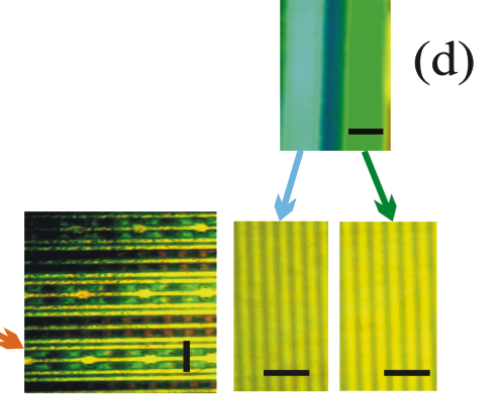

(f)

Figure 5. Images of a CPU: (a) Under conventional white-light illumination; (b), (c) Using spectral encoding - in (c) the sample is rotated counter-clockwise by $90^{\circ}$; (d) A magnified portion of (b); (e), (f) Bright-field reflection microscope images. Image size in (a), (b) and (c) is $15 \times 15$ $\mathrm{mm}$. Scale bar in (d) is $50 \mu \mathrm{m}$ and in (e), (f) is $10 \mu \mathrm{m}$. The colour scale is in $\mu \mathrm{m}$. 
(figure 5(b),(c)). The illumination and collection angles were set to $\theta=15^{\circ}$ and $\alpha=6^{\circ}$ to match the transmitted spatial frequency band to that of the sample. The CPU was rotated counter-clockwise about the optical axis by $90^{\circ}$ between the recording of figure $5(\mathrm{~b})$ and figure $5(\mathrm{c})$, demonstrating a simple means of recording the two-dimensional sample spatial frequency spectrum. Errors in measurement of the rotation (azimuthal) angle $\Delta \varphi$ will result in measurement errors for period $H$ of the sample's periodic structure described by $\Delta H=H((1-\cos \Delta \varphi) / \cos \Delta \varphi)$. For our experiments, this error was less than $5 \mathrm{~nm}$. A magnified portion of a spectrally decoded image is shown in figure 5(d), and the corresponding high-resolution, bright-field reflection image (Nikon ECLIPSE 80i, NA $=0.95$ ) is shown in figure 5(f). Figure 5(e) is the bright-field reflection image corresponding to the portion shown in figure 5(c). The areas corresponding to different microstructures are clearly seen in figures 5(b) and (c). The difference in colour readily apparent in figure 5(d) corresponds to a difference in period of $600 \mathrm{~nm}$, as shown in figure 5(f). For high accuracy absolute measurements, it is necessary to carefully calibrate the wavelength (RGB) response of the CCD camera. To be able to achieve the same discrimination using conventional microscopy, it is necessary to use a high-NA objective lens, which brings with it much tighter constraints on the field of view and working distance.

This practical implementation is readily suited to the quantitative characterization of periodic microstructure, as performed by optical critical dimension (OCD) techniques used in microelectronics inspection [15]. Whereas OCD techniques are based on the wavelength and polarization dependences of spectral reflectance, i.e., zero-order diffraction, and require multiple acquisitions, our imaging approach would use the wavelength-dependence of first-order diffraction to characterize such samples, in a single exposure.

A potentially confounding factor for spectral encoding is a sample's wavelength-dependent absorption. It should be straight forward, however, to account for this by normalization with respect to a zero-order image recorded at normal illumination. A compensatory advantage is that, because of its dependence on diffraction, spectral encoding is not sensitive to sample translations, but only to rotations and deformations, which could be detected with high sensitivity. Because transmission of a sample's spatial frequency spectrum is decoupled from the NA of optical system, spectral encoding could even be performed using a single-mode optical fibre.

Although periodic microstructures are widely relevant, including in the generation of structural colour in the natural world [16], this implementation is not limited to such samples. We have demonstrated that, for a complex centimetre-scale sample consisting of a number of periodic diffracting structures, it is possible (at tens-of-centimetre working distance) to form a quantitative, wide-field image sensitive to nanometre-scale local variations in microstructure. The pure colour produced by such diffraction makes the interpretation of the image straight forward. For non-periodic samples, the spatial frequency spectrum of each image resolution cell will be more complex and wider, along with the corresponding optical spectrum. In this case, the specific spectral decoding implementation presented here would display a colour related to the mean spatial frequency in a given local area. To accurately record the full spatial frequency spectrum, imaging spectroscopy with higher resolution than the simple three-colour version demonstrated here would be required, representing a natural extension to this work. Such information is invaluable, but it still does not permit reconstruction of the microstructure within an image resolution cell. Such formation of fully superresolved images based on spectral en/decoding is possible in principle. It would require reconstruction from a set of complex spatial frequency amplitudes, which could be decoded, e.g., holographically by use of a swept-wavelength source.

To completely characterize the spatial features of a sample, it is necessary to make measurements over the whole azimuthal angular range $0-360^{\circ}$ with a step size that is determined by the required resolution and by the complexity of the sample's microstructure. Useful additional information could be obtained during this process. For example, for local areas of a sample containing periodic structures, the corresponding colours would change during sample rotation, whereas, for local areas with more complicated, arbitrarily oriented structures, the colours would not change significantly. Thus, the 
presence of periodic structure could be simply detected. Simultaneous measurement of portions of the two-dimensional spatial frequency spectrum is also possible, e.g., by using multiple illumination waves at different azimuthal angles and correspondingly orientated decoding diffraction gratings. Such waves could be multiplexed/demultiplexed based on, e.g., polarization.

For the first time to our knowledge, we have demonstrated that spatial frequency-domain optical spectral encoding based on broadband, off-axis plane wave illumination enables the transmission of an instantaneous sample spatial frequency bandwidth greatly exceeding the diffraction limited bandwidth of the optical system used to transmit it. In addition, we have shown that this superresolution approach, when combined with simple spectral decoding, is useful in sample characterization. Even with the modest wavelength sensitivity of the specific spectral decoding scheme employed here, we have demonstrated sub-micron sensitivity in one direction with a single-capture. Such performance demonstrates that the technique is capable of quantitative dynamic imaging and characterization of centimetre-scale samples.

\section{References}

[1] Sheppard C J R 2007 Fundamentals of superresolution Micron 38 165-9

[]2 Gustafsson M 2005 Nonlinear structured-illumination microscopy: Wide-field fluorescence imaging with theoretically unlimited resolution PNAS 102 13081-86

[3] Bossi M, Fölling J, Dyba M, Westphal V and Hell S W, 2006 Breaking the diffraction resolution barrier in far-field microscopy by molecular optical bistability New J. Phys. 8275

[4] Kartashev A I 1960 Optical systems with enhanced resolving power Opt. Spectrosc. 9 204-6

[5] Leith E N, Angell D, and Kuei C-P 1987 Superresolution by incoherent-to-coherent conversion J Opt. Soc. Am. A 4 1050-4

[6] Mico V, Zalevsky Z, Garcia-Martinez P and Garcia J 2006 Superresolved imaging in digital holography by superposition of tilted wavefronts Appl. Opt. 45 822-8

[7] Alexandrov S A, Hillman T R, Gutzler T and Sampson D D, 2006 Synthetic aperture Fourier holographic optical microscopy Phys. Rev. Lett. 97168102

[8] Gurjar R S, Backman V, Perelman L T, Georgakoudi I, Badizadegan K, Itzkan I, Dasari R R and Feld M S 2001 Imaging human epithelial properties with polarized light-scattering spectroscopy Nature Med. $71245-8$

[9] Hillman T R, Alexandrov S A, Gutzler T and Sampson D D 2006 Microscopic particle discrimination using spatially-resolved Fourier-holographic light scattering angular spectroscopy Opt. Express 1411088 102

[10]Hillman T R, Adie S G, Seemann V, Armstrong J J, Jacques S L and Sampson D D 2006 Correlation of static speckle with sample properties in optical coherence tomography Opt. Lett. 31 190-2

[11]Mico V, Garcia J, Ferreira C, Sylman D and Zalevsky Z 2007 Spatial information transmission using axial temporal coherence coding Opt. Lett. 32 736-8

[12]Paek E G, Zah C E, Cheung K W, and Curtis L 1992 All-optical image transmission through a single mode fiber Opt. Lett. 17 613-5

[13]Mendlovic D, Garcia J, Zalevsky Z, Marom E, Mas D, Ferreira C and Lohmann A W 1997

Wavelength-multiplexing system for single-mode image transmission Appl. Opt. 36 8474-80

[14]Goodman J W, Introduction to Fourier optics (McGraw-Hill, New York, 1996, 2nd ed.)

[15]Hoobler R and Gise P 2005 Mask metrology using OCD for profiling Solid State Technol. 48 35-8

[16]Vukusic P and Sambles J R 2003 Photonic structures in biology Nature 424 852-5 